\title{
Artificial Intelligence System Application in Miliary Lung Metastasis: Experience from a Rare Case
}

\author{
Yu Zhang ${ }^{1, *}$ \\ Yan Chen ${ }^{2, *}$ \\ Kun $\mathrm{Li}^{2}$ \\ Wen Jiang ${ }^{3}$ \\ Bi-Cheng Zhang ${ }^{4}$ \\ 'Department of Pathology, General \\ Hospital of Central Theater Command of \\ the People's Liberation Army, Wuhan, \\ 430070, People's Republic of China; \\ ${ }^{2}$ Department of Anesthesiology, General \\ Hospital of Central Theater Command of \\ the People's Liberation Army, Wuhan, \\ 430070, People's Republic of China; \\ ${ }^{3}$ Department of Health Medicine, \\ General Hospital of Central Theater \\ Command of the People's Liberation \\ Army, Wuhan, 430070, People's Republic \\ of China; ${ }^{4}$ Cancer Center, Renmin \\ Hospital of Wuhan University, Wuhan, \\ 430060, People's Republic of China
}

*These authors contributed equally to this work
Correspondence: Bi-Cheng Zhang Cancer Center, Renmin Hospital of Wuhan University, 99\#, Zhangzhidong Road, Wuchang District, Wuhan, Hubei, 430060, People's Republic of China Tel +86-1387I000940

Email bichengzhang@hotmail.com

Wen Jiang

Department of Health Medicine, General Hospital of Central Theater Command of the People's Liberation Army, 627\#,

Wuluo Road, Wuchang District, Wuhan, Hubei, 430070, People's Republic of China Fax +86-27-50772388

Email zbzqzyyjw@I63.com
Introduction: Miliary intrapulmonary carcinomatosis (MIPC) is very rare in the existing literature. We reported a lung adenocarcinoma patient presented with over 200 uniform size pulmonary nodules in all lung lobes at the initial examination. The application of artificial intelligence (AI) in lung cancer has been gradually reported, but not yet reported in MIPC. The application of AI in this rare disease is worth exploring.

Patient Information: A 57-year-old woman received chest computed tomography (CT) scan because of dry cough, intermittent chest wall and back pain for 3 weeks. CT imaging found over 200 uniform size pulmonary nodules in an evenly dispersed pattern at bilateral lungs with a $38 \times 45 \mathrm{~mm}$ new creature at the dorsal segment of the lower lobe of the left lung. However, as a very reliable diagnostic assistant system in CT imaging of lung cancer, AI can only identify 18 nodules in such classic metastatic lung cancer case.

Conclusion: This case provides classical imaging figures as textbook-like, even though there is no such classic imaging of lung metastases in the existing textbooks. This medical imaging material will impress medical students and help them learn about the disease deeply. This medical imaging material can warn patients to recognize the horror of lung cancer metastasis and has good popularization of science. This medical imaging material presents a new challenge for AI.

Keywords: miliary nodules, lung adenocarcinomas, metastases, artificial intelligence

\section{Introduction}

Lung cancer is the leading cause of cancer death with the highest cancer morbidity worldwide. ${ }^{1}$ The primary cause of death is metastases but not the primary tumor. ${ }^{2}$ The common metastatic pathway includes the local tumor invasion into neighboring tissues, lymphatic system, and hematogenous system. Miliary intrapulmonary carcinomatosis (MIPC) is an uncommon phenomenon that results from lung cancer cells' hematogenous dissemination. Here we present a classic case of lung cancer patients with miliary lung metastasis at the time of the first diagnosis. It is a new system with proven high differential diagnosis of lung cancer or pneumonia in the existing literature, but not yet reported in MIPC. The application of AI in this rare disease is worth exploring.

\section{Case Report}

A 57-year-old woman presented to our hospital because of dry cough, intermittent chest wall and back pain for 3 weeks. She complained the pain gradually aggravated but did not influence the length of sleep bouts. Simultaneously, the level of daily physical activity was significantly decreased, with a $3 \mathrm{~kg}$ unintentional weight 
loss over the past month. She denied tobacco and tuberculosis exposure history. Physical examination suggests obvious swollen bilateral supraclavicular lymph nodes.

A chest enhanced computed tomography (CT) scan revealed over 200 uniform size pulmonary nodules in an evenly dispersed pattern at bilateral lungs with a $38 \times 45 \mathrm{~mm}$ new creature at the dorsal segment of the lower lobe of the left lung (Figure $1 \mathrm{~A}-\mathrm{C}$ ), in addition to a small amount of left-sided pleural and pericardial effusion. Interestingly, the artificial intelligence (AI) system (The AI software supported by Yitu CT, YITU Healthcare Technology Co., Ltd., China. The AI system of deep learning-based quantification was performed using a novel established lung nodule net based on one automatic segmentation software. This module was developed as a combination of U-net and fully convolutional networks. Approaches for lung nodule segmentation involved the detection of a volume of interest (VOI) covering the nodule area and segmentation inside this VOI., ${ }^{3,4}$ These methods can be generally classified into machine-learning methods based on China big date, which covered more than 10 provinces and more than 50 hospitals), only identified 18 pulmonary nodules (Figure 2), which are lower than $10 \%$ of the real data. Besides, all of these were judged as low-risk nodules. Another enhanced CT scans showed multiple liver and adrenal metastases, multiple bone metastases (including thoracic spine, sternum, ribs and left scapula), as well as apparent lymphadenopathy in the left hilar, bilateral mediastinal and retroperitoneal area. Magnetic resonance imaging (MRI) revealed multiple osteolytic bone destruction in thoracic vertebrae and a T7 vertebral body's compression fracture. T-SPOT was negative while a significant elevation of the serum tumor markers (including CEA $839.30 \mathrm{ng} / \mathrm{mL} \uparrow$, NSE

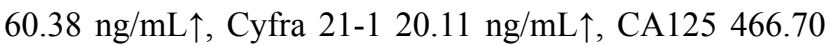
$\mathrm{U} / \mathrm{mL} \uparrow, \mathrm{CA} 153181.00 \mathrm{U} / \mathrm{mL} \uparrow, \mathrm{CA} 19974.16 \mathrm{U} / \mathrm{mL} \uparrow$ and CA724 $151.40 \mathrm{U} / \mathrm{mL} \uparrow)$. Biopsy of the left supraclavicular node confirmed metastatic adenocarcinoma (Figure 3A) with supportive immunohistochemical staining reaction: positive for TTF-1 (Figure 3B), PCK (Figure 3C), CK7, but negative for P40, Syn, and the Ki 67 index was reported in $70 \%$ (Figure 3D). The peripheral blood gene detection showed wild-type epidermal growth factor receptor (EGFR) with no gene mutation sites. In light of

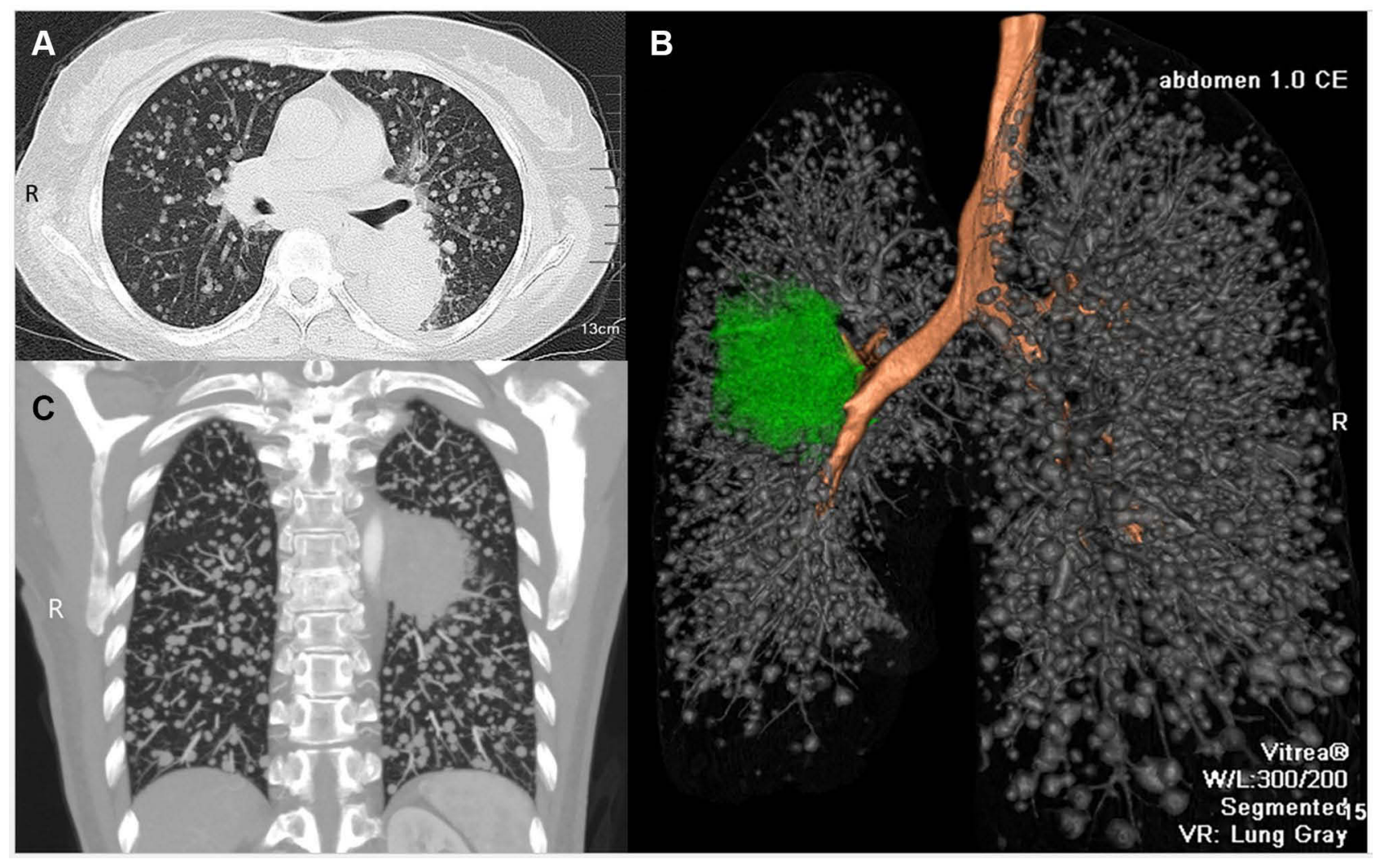

Figure I 16-Slice computed tomography scans during initial diagnosis. The axial planes (A), 3D view (B), coronal planes (C) showed a blocky mass in the left lung and uniform miliary nodules dispersed all lung lobes. 


\section{Al system analysis result}

\begin{tabular}{|c|c|c|}
\hline $\begin{array}{l}\text { Right upper lobe } \\
\text { Nodules: } 3\end{array}$ & $\begin{array}{l}\text { Left upper lobe } \\
\text { Nodules: } 3\end{array}$ & $\begin{array}{l}\text { Gender: Female } \\
\text { Age: } 57 \text { years } \\
\text { Equipment type: TOSHIBA-aquilion } \\
\text { Examine no.: } 20200806001641 \\
\text { CT scan thickness: } 7.00 \mathrm{~mm}\end{array}$ \\
\hline $\begin{array}{l}\text { Right middle lobe } \\
\text { Nodules: } 2\end{array}$ & & $\begin{array}{ll}\text { High-risk nodules } \quad 1 \\
\text { Intermediate-risk nodules }\end{array}$ \\
\hline $\begin{array}{l}\text { Right lower lobe } \\
\text { Nodules: } 6\end{array}$ & $\begin{array}{l}\text { Left lower lobe } \\
\text { Nodules: } 4\end{array}$ & \\
\hline
\end{tabular}

Figure 2 Artificial intelligence (Al) system (the Al software supported by Hangzhou Yitu Medical Technology Limited Company) identified one high-risk nodules and I7 lowrisk nodules.

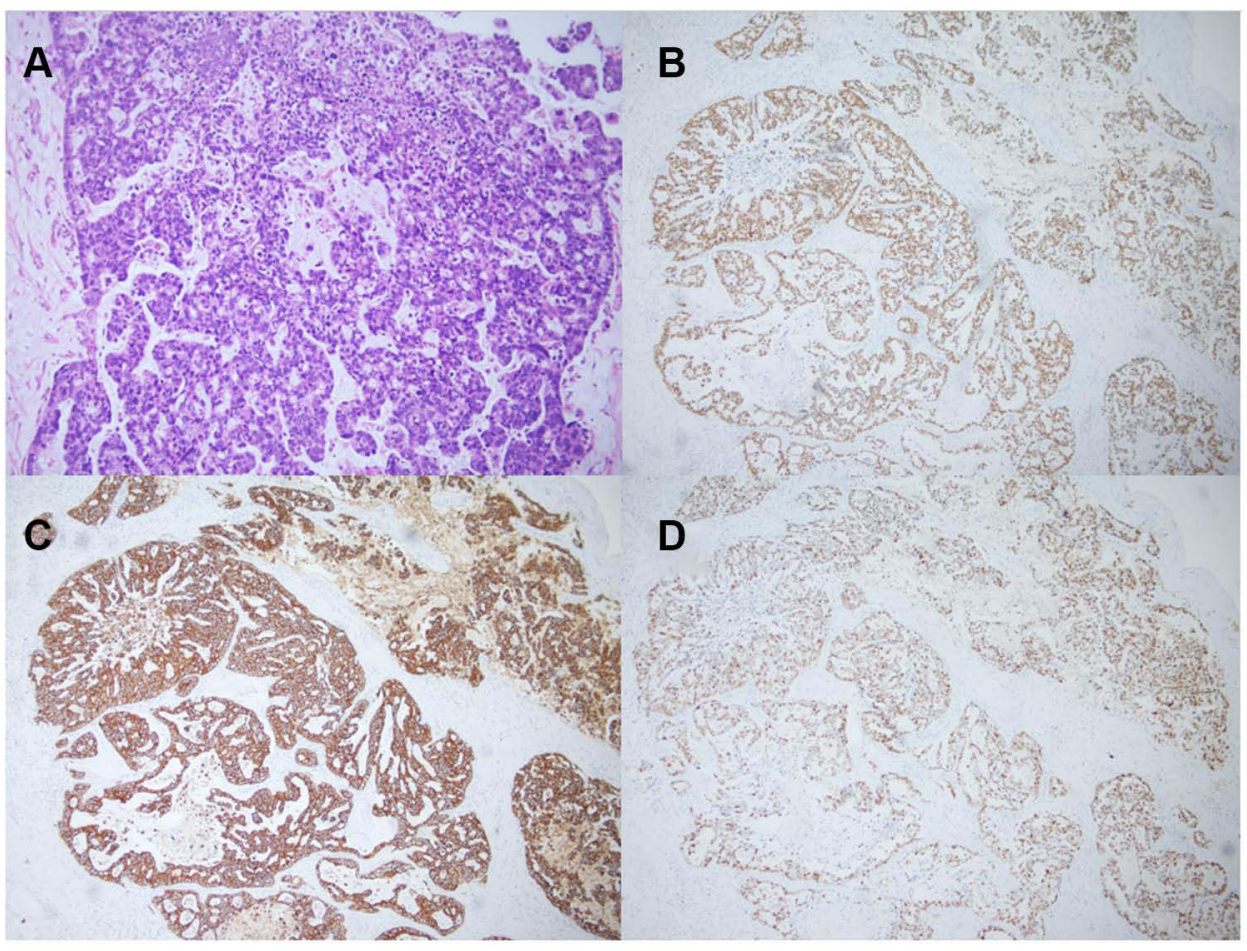

Figure 3 Image of histologic diagnosis using hematoxylin and eosin staining (A) (original $\times 100$ magnification) from the enlarged left supraclavicular lymph nodes at the time of the initial diagnosis metastatic lung adenocarcinoma. Immunohistochemistry of TTF-I (B), PCK (C), and Ki-67 (D) (original $\times 100$ magnification).

clinical and instrumental examinations, the diagnosis of lung adenocarcinoma (T4N3M1 stage IV), EGFRnegative, was made.

This patient lost the chance of surgical treatment due to the extensive metastatic transfer of primary tumors to distant organs. The negative gene detection result indicated that she could not benefit from target therapy. We recommended a combination therapy regimen, the subsequent imaging revealed a significant decrease in the size and number of primary tumors and metastases (Figure 4). 


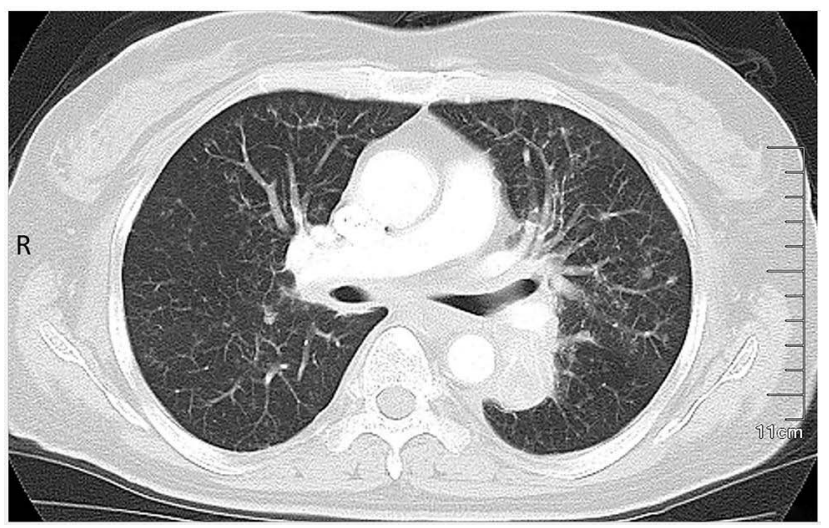

Figure 4 After combined therapy, the primary tumor size significantly decreased and the miliary lung metastasis almost wholly disappeared on I6-slice computed tomography.

\section{Discussion}

As shown in this report, the axial and coronal view CT examination revealed a homogeneously distributed pattern of miliary nodules in all lobes of the left and right lungs. The three-dimensional (3D) CT reconstruction further revealed the stereometric and 3D structure of all nodules and the relationship between vessels and the distribution of the nodules in the lung lobes. In clinical practice, the number of lung cancer metastases in the lung is usually only a few or 10 to dozens of lesions. Miliary intrapulmonary carcinomatosis (MIPC) is an uncommon phenomenon that results from lung cancer cells' hematogenous dissemination. There are only few reports on the occurrence of MIPC in the existing literature. ${ }^{5-7}$

MICP is characterized by uncountable discrete pulmonary micronodules, generally tiny and uniform in size ( $\leq 5 \mathrm{~mm}$ ) and diffusely distributed throughout the lungs. ${ }^{8,9}$ MICP may be predictive for rapid tumor progress. ${ }^{10}$ MIPC is sporadic in clinical practices that could easily be misdiagnosed because miliary nodules are generally acknowledged as an imaging manifestation of disseminated tuberculosis. Additionally, sarcoidosis, silicosis, extrathoracic malignancy histoplasmosis were relatively uncommon etiologies of miliary nodules. ${ }^{11}$ The differential diagnosis of lung cancer from other lung diseases is always worth studying and exploring. ${ }^{12,13}$ Detailed clinical history, laboratory findings and histopathological examination are essential to confirm the diagnosis.

AI adopting deep learning technology has been widely used in the medical imaging domain in recent years. ${ }^{14} \mathrm{AI}$ will take a pivotal position in lung cancer diagnosis with chest CT images because of the high accuracy (90\%) on classification with a sensitivity of $81 \%$ and specificity of $95 \% .{ }^{15}$ However, there is a huge gap between the AI interpretation results and the actual situation in our case. The possible reasons are 1) this case is rare worldwide and has not been learned by AI system previous; 2) there are too many metastatic nodules; and 3) the AI system needs to be further corrected and improved in these kinds of figures.

In short, this case has shown significant and practical value for clinical teaching and fits as textbook models. These figures would impress upon the beginners or experts in the academic field, immensely help them keep in mind the classic images of lung metastases. It would also increase the patients' and families' awareness and understanding of lung cancer and lung metastases. Last, but not least, this medical imaging material presents a new challenge for AI systems.

\section{Ethics}

The authors are accountable for all aspects of the work in ensuring that questions related to the accuracy or integrity of any part of the work are appropriately investigated and resolved. The study was conducted in accordance with the Declaration of Helsinki (as revised in 2013).

\section{Informed Consent for Publication}

Written informed consent was obtained from the patient for the publication. The patient provided written informed consent to participate in this study.

\section{Acknowledgment}

These authors contributed equally to this work and should be considered as co-first authors: Yan Chen and Yu Zhang. 


\section{Disclosure}

The authors declare that they have no conflicts of interest for this work.

\section{References}

1. Bray F, Ferlay J, Soerjomataram I, Siegel RL, Torre LA, Jemal A. Global cancer statistics 2018: GLOBOCAN estimates of incidence and mortality worldwide for 36 cancers in 185 countries. CA Cancer J Clin. 2018;68(6):394-424. doi:10.3322/caac.21492

2. Bailey PC, Martin SS. Insights on CTC biology and clinical impact emerging from advances in capture technology. Cells. 2019;8(6):553. doi:10.3390/cells8060553

3. Wang S, Zhou M, Liu Z, et al. Central focused convolutional neural networks: developing a data-driven model for lung nodule segmentation. Med Image Anal. 2017;40:172-183. doi:10.1016/j. media.2017.06.014

4. Pan F, Li L, Liu B, et al. A novel deep learning-based quantification of serial chest computed tomography in Coronavirus disease 2019 (COVID-19). Sci Rep. 2021;11(1):417. doi:10.1038/s41598-02080261-w

5. Otoshi R, Sekine A, Okudela K, et al. Miliary lung metastases from ROS1-rearranged lung adenocarcinoma: a case report. Mol Clin Oncol. 2020;13(1):80-82. doi:10.3892/mco.2020.2040

6. Kurihara M, Koda H, Aono H, et al. Rapidly progressive miliary brain metastasis of lung cancer after EGFR tyrosine kinase inhibitor discontinuation: an autopsy report. Neuropathology. 2019;39(2):147-155. doi:10.1111/neup. 12542

7. Pillai S, Khan A, Khan S. Adenocarcinoma of the lung presenting with intrapulmonary miliary metastasis. Cureus. 2019;11(8):e5430.
8. Andreu J, Mauleón S, Pallisa E, Majó J, Martinez-Rodriguez M, Cáceres J. Miliary lung disease revisited. Curr Probl Diagn Radiol. 2002;31(5):189-197. doi:10.1067/mdr.2002.127634

9. Kim HJ, Kang SH, Chung HW, et al. Clinical features of lung adenocarcinomas with epidermal growth factor receptor mutations and miliary disseminated carcinomatosis. Thorac Cancer. 2015;6 (5):629-635.

10. Lim CK. The moth-eaten lung: lung adenocarcinoma with cavitating miliary intrapulmonary carcinomatosis. J Thorac Oncol. 2015;10 (9):1375. doi:10.1097/JTO.0000000000000542

11. Salahuddin M, Karanth S, Ocazionez D, Estrada-Y-Martin RM, Cherian SV. Clinical characteristics and etiologies of miliary nodules in the US: a single-center study. Am J Med. 2019;132(6):767-769. doi:10.1016/j.amjmed.2018.12.030

12. Zhu J, Zhang Y, Gao XH, Xi EP. Coronavirus disease 2019 or lung cancer: a differential diagnostic experience and management model from Wuhan. $J$ Thorac Oncol. 2020;15(8):e141-e142. doi:10.1016/j. jtho.2020.04.030

13. Zhu J, Huang WC, Huang B, et al. Clinical characteristics and prognosis of COVID-19 patients with initial presentation of lung lesions confined to a single pulmonary lobe. Am J Transl Res. 2020;12(11):7501-7509.

14. Baldwin DR, Gustafson J, Pickup L, et al. External validation of a convolutional neural network artificial intelligence tool to predict malignancy in pulmonary nodules. Thorax. 2020;75(4):306-312. doi:10.1136/thoraxjnl-2019-214104

15. Ren Y, Tsai MY, Chen L, et al. A manifold learning regularization approach to enhance 3D CT image-based lung nodule classification. Int J Comput Assist Radiol Surg. 2020;15(2):287-295. doi:10.1007/ s11548-019-02097-8
Risk Management and Healthcare Policy

\section{Publish your work in this journal}

Risk Management and Healthcare Policy is an international, peerreviewed, open access journal focusing on all aspects of public health, policy, and preventative measures to promote good health and improve morbidity and mortality in the population. The journal welcomes submitted papers covering original research, basic science, clinical \& epidemiological studies, reviews and evaluations, guidelines, expert opinion and commentary, case reports and extended reports. The manuscript management system is completely online and includes a very quick and fair peer-review system, which is all easy to use. Visit http://www.dovepress.com/testimonials.php to read real quotes from published authors. 